VOL. $72(2005) \quad[1-6]$

\title{
A NOTE ON THE LATTICE OF DENSITY PRESERVING MAPS
}

\author{
Sejal Shah and T.K. Das
}

\begin{abstract}
We study here the poset $D P(X)$ of density preserving continuous maps defined on a Hausdorff sapce $X$ and show that it is a complete lattice for a compact Hausdorff space without isolated points. We further show that for countably compact $T_{3}$ spaces $X$ and $Y$ without isolated points, $D P(X)$ and $D P(Y)$ are order isomorphic if and only if $X$ and $Y$ are homeomorphic. Finally, Magill's result on the remainder of a locally compact Hausdorff space is deduced from the relation of $D P(X)$ with posets $I P(X)$ of covering maps and $E_{K}(X)$ of compactifications respectively.
\end{abstract}

\section{INTRODUCTION}

Throughout the spaces considered (usually denoted by symbols $X, Y$ ) are Hausdorff and the maps are continuous. A map $f: X \rightarrow Y$ is called a density preserving map if Int $\mathrm{Cl} f(A) \neq \phi$, whenever $\operatorname{Int} A \neq \phi, A \subseteq X$ ([1]). Two density preserving maps $f$ and $g$ with domain $X$ and range $R f$ and $R g$ respectively are said to be equivalent $(f \approx g)$ if there exists a homeomorphism $h: R f \rightarrow R g$ satisfying $h \circ f=g$. We identify equivalent density preserving maps on a fixed domain $X$, and denote by $D P(X)$ the set of all such equivalent classes of density preserving maps. The relation ' $\leqslant$ ' defined on $D P(X)$ by $g \leqslant f$ if there exists a continuous map $h: R f \rightarrow R g$ such that $h \circ f=g$ turns out to be a partial order relation. Recall that a perfect irreducible continuous surjection is called a covering map. In Section 1 we prove that if $X$ is a compact space without isolated points, then $D P(X)$ is a complete lattice. In Section 2, we determine the order structure of $D P(X)$ by proving that for countably compact $T_{3}$ spaces $X$ and $Y$ without isolated points, $D P(X)$ and $D P(Y)$ are order isomprphic if and only if $X$ and $Y$ are homeomorphic. Section 3 is devoted to the natural relation of $D P(X)$ with the poset $I P(X)$ of covering maps on $X([3])$ and the poset $E_{K}(X)$ of compactifications of a locally compact space $X$ ([2]). We show that if $U$ is an open dense set in a compact space $X$ then $D P(X, U)=I P(X, U)$, where $I P(X, U)$ (respctively $D P(X, U)$ ) is the poset of all covering (respectively density preserving-) maps $f$ on $X$ satisfying $\left|f^{-1}(f(x))\right|=1$ for each $x$ in $U$. Using this result we deduce Magill's result which states that for locally compact spaces $X$ and $Y, E_{K}(X)$ and $E_{K}(Y)$ are order isomorphic if and only if $\beta X-X$ and $\beta Y-Y$ are homeomorphic ([2]).

Received 12th January, 2005

Copyright Clearance Centre, Inc. Serial-fee code: 0004-9727/05 \$A2.00+0.00. 


\section{LATTICE $D P(X)$}

We immediately have the following lemmas.

LEMMA 1.1. $D P(X)$ is a partially ordered set.

LEMMA 1.2. Let $f, g \in D P(X)$ be such that $g \leqslant f$. Then the map $h: R f$ $\rightarrow R g$ satisfying $h \circ f=g$ is a density preserving map.

Proof: Let $A \subseteq R f$ be such that Int $A \neq \phi$. Then by setting $f^{-1}(A)=A^{*}$, we get $\phi \neq \operatorname{Int} \mathrm{Cl} g\left(A^{*}\right)=\operatorname{Int} \mathrm{Cl}(h \circ f)\left(A^{*}\right) \subseteq \operatorname{Int} \mathrm{Cl} h(A)$. Hence $h$ is a density preserving map.

REMARK 1.3. Fibres of a surjective density preserving map $f: X \rightarrow Y$ are closed nowhere dense subsets of $X$, where $X$ is a space without isolated points.

Definition 1.4: For $f \in D P(X)$, define $\wp(f)=\left\{f^{-1}(y) \mid y \in R f\right\}$.

From here onwards we assume that members of $D P(X)$ are quotient maps. If $X$ is compact, this condition is automatically satisfied.

LEMMA 1.5. Let $f, g \in D P(X)$. Then $f \leqslant g$ if and only if $\wp(g) \subseteq \wp(f)$.

Proof: Let $f \leqslant g$ then there exists $h: R g \rightarrow R f$ satisfying $h \circ g=f$. If $g^{-1}(y)=A \in \wp(g)$ and if $h(y)=x$, then $A \subseteq(h \circ g)^{-1}(x)=f^{-1}(x)$. Conversely, suppose $\varphi(g) \subseteq \wp(f)$, then for $z \in R g$ take the unique $y \in R f$ for which $g^{-1}(z)$ $\subseteq f^{-1}(y)$ and define $h: R g \rightarrow R f$ by $h(z)=y$. Clearly $h$ is continuous, $h \circ g=f$ and hence $f \leqslant g$.

Note 1.6. Two maps $f$ and $g$ are equivalent if and only if $\wp(f)=\wp(g)$.

LEMMA 1.7. Let $X$ be a compact space without isolated points. Then $D P(X)$ is a complete upper semi-lattice.

Proof: Let $S$ be a non-empty subset of $D P(X)$ and let $Z=\prod\{R f \mid f \in S\}$. Consider the natural evaluation map $g: X \rightarrow Z$ such that $\pi_{f}(g(p))=f(p)$, where $\pi_{f}: Z \rightarrow R f$ is the $f^{\text {th }}$ projection map. Set $T=g(X), \pi_{f}^{\prime}=\left.\pi_{f}\right|_{T}$ and define $g^{\prime}: X \rightarrow T$ by $g^{\prime}(p)=g(p), p \in X$. It is easy to verify that $g^{\prime}$ is the least upper bound of $S$.

TheOREM 1.8. Let $X$ be a compact space without isolated points. Then $D P(X)$ is a complete lattice.

PROOF: Since a constant map onto its image is a density preserving map and any two such maps are equivalent, $D P(X)$ has the minimum element. The required result now follows from Lemma 1.7 and the fact that a complete upper semilatice with minimum element is a complete lattice. 


\section{Order Structure of $D P(X)$}

The order structure of the poset $D P(X)$ is always determined by the topology on $X$, that is, if spaces $X$ and $Y$ are homeomorphic then $D P(X)$ and $D P(Y)$ are order isomorphic. We show here that the converse is true when $X$ and $Y$ are countably compact $T_{3}$ spaces without isolated points. The following terms and results are along the lines of $[2$, Lemmas 6,9 and 10]. Throughout this section, our spaces are without isolated points.

Definition 2.1: A Map $f \in D P(X)$ is said to be

(i) primary if $\wp(f)$ has at most one non-singleton member.

(ii) dual if it is primary and $\wp(f)$ contains exactly one doubleton.

Notation. If for some $f \in D P(X), \wp(f)$ contains $n$ non-singleton members, say $K_{1}, K_{2}, \ldots, K_{n}$, then $f$ is denoted by $\left(f, K_{1}, K_{2}, \ldots, K_{n}\right)$. In particular, if $K$ is a non-singleton closed nowhere dense set in $X$, then $(f, K)$ denotes the natural density preserving map defined on $X$ obtained by collapsing $K$ to a point.

\section{LEMMA 2.2.}

I $A$ map $f \in D P(X), f \neq i d_{X}$ is primary (respectively dual) if and only if there do not exist dual points $g, h \in D P(X)$ (respectively $g \in D P(X)$ ) such that $f \wedge g=f \wedge h \neq f$ and the only dual points greater than $g \wedge h$ are $g$ and $h$ (respctively $f<g<i d_{X}$ ).

II For two closed nowhere dense subsets $K_{1}$ and $K_{2}$ of $X$,

$$
\left(f, K_{1}\right) \wedge\left(g, K_{2}\right)= \begin{cases}\left(h, K_{1}, K_{2}\right), & \text { if } K_{1} \cap K_{2}=\phi \\ \left(h, K_{1} \cup K_{2}\right), & \text { if } K_{1} \cap K_{2} \neq \phi .\end{cases}
$$

III An oder isomorphism $\varphi: D P(X) \rightarrow D P(Y)$ maps dual points to dual points.

Definition 2.3: A bijection $f: X \rightarrow Y$ is called a cln-bijection if $\{f(A) \mid A$ is a closed nowhere dense subset of $X\}=\{B \mid B$ is closed nowhere dense subset of $Y\}$.

LEMmA 2.4. Let $\varphi: D P(X) \rightarrow D P(Y)$ be an order isomorphism. Then there exists a cln-bijection $F: X \rightarrow Y$ such that $f \in D P(X)$ implies $\wp(\varphi(f))=\{F(A) \mid$ $A \in \wp(f)\}$.

ProOF: Take $p \in X$ and choose distinct points $q, r \in X-\{p\}$. By Lemma 2.2(III), $\varphi(f,\{p, q\}), \varphi(g,\{p, r\})$ are dual points of $D P(Y)$ say $(\bar{f},\{a, b\})$ and $(\bar{g},\{c, d\})$ respectively. Clearly $(\bar{f},\{a, b\}) \wedge(\bar{g},\{c, d\})=\varphi(f \wedge g,\{p, q, r\})$. If $\{a, b\} \cap$ $\{c, d\}=\phi$, then $(\bar{f},\{a, b\}) \wedge(\bar{g},\{c, d\})=(\bar{f} \wedge \bar{g},\{a, b\},\{c, d\}) ;(f,\{p, q\}),(g,\{p, r\})$, $(h\{q, r\})$ are three dual points greater than $(f \wedge g,\{p, q, r\})$ and $(f,\{a, b\}),(\bar{g},\{c, d\})$ 
are two dual points greater than $(\bar{f} \wedge \bar{g},\{a, b\},\{c, d\})$ which is not possible. Therefore $\{a, b\} \cap\{c, d\} \neq \phi$, in fact it is a singleton, say $\{a\}$. Define $F: X \rightarrow Y$ by $F(p)=a$. Note that the choice of $a$ does not depend on the choice of $r$ and $q$. In general, if $f \in D P(X)$ is of the form $(f, H)$ and if $\varphi(f, H)=\bar{f}$, then it is easy to verify that $\bar{f}=(\bar{f}, K)$ for some closed nowhere dense subset $K$ of $Y$. Further, if $p, q \in H, p \neq q$ then $(g,\{p, q\}) \geqslant(f, H)$ which implies $(\bar{g},\{a, b\}) \geqslant(\bar{f}, K)$ therefore $F(\{p, q\})=\{a, b\} \subseteq K$ and hence $F(H) \subseteq K$. Similarly we can use $\varphi^{-1}$ to define $\bar{F}: Y \rightarrow X$ and obtain $\bar{F}(K) \subseteq H$. Observe that $\bar{F} \circ F$ is identity on $X$. In fact, if $p \in X$ and $q \in X-\{p\}$, then $\varphi(f,\{p, q\})$ is dual point say $(f,\{a, b\})$ and $F(p) \in\{a, b\}$. Assume $F(p)=a$. Suppose $\bar{F}(a) \neq p$. Then $\bar{F}(a)=q$. Choose $r \in X-\{q, p\}$ then there exists $c \in Y$ such that $\varphi(g,\{p, r\})$ is a dual point say $(\bar{g},\{a, c\})$. Since $\bar{F}(a) \in\{p, r\}$ and $\bar{F}(a) \neq p$, therefore $\bar{F}(a)=r$, a contradiction. Similaraly, $F \circ \bar{F}$ is identity on $Y$. We have also shown in the process that if $\varphi(f, H)=(f, K)$, then $F(H)=K$.

Recall that a subset $A$ of countably compact $T_{3}$ space $X$ without isolated points is closed if and only if whenever $B \subseteq A$ and $\mathrm{Cl}_{X} B$ is nowhere dense in $X$ then $\mathrm{Cl}_{X} B \subseteq A$. Using this fact, Lemma 2.4 and the technique of [3, Theorem 1.1], we have the following.

THEOREM 2.5. Let $X$ and $Y$ be countably compact $T_{3}$ spaces without isolated points. Then $D P(X)$ and $D P(Y)$ are order isomorphic if and only if $X$ and $Y$ are homeomorphic.

Note 2.6. The map $f: Q \cup\{p\} \rightarrow Q \cup\{q\}$ in [3, example 3.9] defined by $f(x)=x$ if $x \in Q$ and $f(p)=q$, where $p$ and $q$ are remote points of $Q$ such that Stone's extension of no self-homeomorphism of $Q$ maps $p$ to $q$, is a cln-bijection between non countably compact spaces which is not a homeomorphism.

\section{3. $D P(X)$ AND $I P(X)$}

Definition 3.1: For a subset $A$ in $X$ we define

$$
D P(X, A)=\left\{f \in D P(X)|| f^{-1}(f(x)) \mid=1, \text { for all } x \in A\right\} .
$$

NOTE 3.2.

(i) $D P(X, A)$ is a poset with respect to the order defined on $D P(X)$.

(ii) If $g \in D P(X, A), f \in D P(X)$ and $g \leqslant f$, then $f \in D P(X, A)$.

TheOREM 3.3. Let $A$ be a subset of a compact space $X$ containing all isolated points of $X$. The $D P(X, A)$ is a complete upper semilattice.

Proof: Follows from Lemma 1.7 and Note 3.2(ii). 
THEOREM 3.4. Let $A_{i}$ be any subset of $X_{i}$ containing all isolated points of $X_{i}$, $i=1,2$ and $\varphi: D P\left(X_{1}, A_{1}\right) \rightarrow D P\left(X_{2}, A_{2}\right)$ be an order isomorphism. Then there is a cln-bijection $F: X_{1}-A_{1} \rightarrow X_{2}-A_{2}$.

Proof: Follows along the lines of Lemma 2.4.

THEOREM 3.5. Let $A$ be a dense subspace of a space $X$. Then every $f$ in $D P(X, A)$ is ireducible.

Proof: Let $f \in D P(X, A) . F$ be a proper closed subset of $X$ and $f(F)=R f$. Then for every $y \in(X-F) \cap A,\left|f^{-1}(f(y))\right| \neq 1$ which contradicts the choice of $f$.

Corollary 3.6. If $X$ is compact and $A$ is dense in $X$ then $D P(X, A)$ $=I P(X, A)$. In particular, if $X$ is locally compact the $D P(\alpha X, X)=I P(\alpha X, X)$, where $\alpha X$ is a compactification of $X$.

Proof: Set $D_{C}(X, A)=\{f \in D P(X, A) \mid f$ is closed $\}$. Observe that $D_{C}(X, A)$ $\subseteq I P(X)$ and $D_{C}(X, A)=D P(X, A)$.

Note 3.7. In general, if $A$ is not dense then $D_{C}(X, A) \subseteq I P(X)$ need not be true. For example take $X=[0,1], A=[0,1 / 2)$ and define $f: X \rightarrow X$ by

$$
f(x)=\left\{\begin{array}{ll}
2 x, & 0 \leqslant x \leqslant \frac{1}{2} \\
\frac{3}{2}-x, & \frac{1}{2} \leqslant x \leqslant 1
\end{array} . \text { Clearly } f \in D_{C}(X, A)-I P(X) .\right.
$$

We recall the following result [3, Lemma 3.11].

LEMMA 3.8. Let $X$ be a locally compact space. The function $\psi: I P(\beta X, X)$ $\rightarrow E_{K}(X)$ defined by $\psi(f)=\beta X \mid \wp(f)$ is an order isomorphism, where $\beta X \mid \wp(f)$ is the natural compactification of $X$ obtained by collapsing each fibre in $\wp(f)$ to a point.

We now deduce following result due to Magill [2, Theorem 12].

THEOREM 3.9. Let $X$ and $Y$ be locally compact spaces. Then $E_{K}(X)$ and $E_{K}(Y)$ are order isomorphic if and only if $\beta X-X$ and $\beta Y-Y$ are homeomorphic.

PRoOF: If $E_{K}(X)$ and $E_{K}(Y)$ are ordered isomorphic, then by Corollary 3.6 and Lemma 3.8, $D P(\beta X, X)$ and $D P(\beta Y, Y)$ are order isomorphic and hence Theorem 3.4 gives a cln-bijection $F: \beta X-X \rightarrow \beta Y-Y$. Since all closed subsets in $\beta X-X$ are nowhere dense, $F$ is a closed map. Similarly $F^{-1}$ is also a closed map.

\section{REFERENCES}

[1] T. Das, 'On projective lift and orbit spaces', Bull. Austral. Math. Soc. 50 (1994), 445-449.

[2] K. Magill, 'The lattice of compactifications of a locally compact space', Proc. London Math. Soc. 28 (1968), 231-244. 
[3] J. Porter and R. Woods, 'The poset of perfect irreducible images of a space', Canad. J. Math. 41 (1989), 193-212.

Department of Mathematics

Faculty of Science

The M.S. University of Baroda

Vadodara - 390002

India

e-mail: skshah2002@yahoo.co.in

tarunkd@yahoo.com 